www.jmscr.igmpublication.org

Impact Factor 5.84

Index Copernicus Value: 83.27

ISSN (e)-2347-176x ISSN (p) 2455-0450

crossref DOI: _https://dx.doi.org/10.18535/jmscr/v5i3.35

Journal Of Medical Science And Clinical Research

IGM Publication

An Official Publication of IGM Publication

\title{
In Vitro Study Comparing the Efficacy of Radiograph and Apex Locator in Determining Working Length in Human Maxillary Molars
}

\author{
Authors \\ Dr Priyadarshini Shetty ${ }^{1}$, Dr Dinesh Shetty MDS $^{2}$, Dr Leena Padhye MDS \\ ${ }^{1}$ MDS, Professor, Dept. of Conservative Dentistry \& Endodontics, Dr. D. Y Patil School of Dentistry, Nerul, \\ Navi Mumbai \\ Ph: 9930155591, Email: drpriyadarshini78@gmail.com \\ ${ }^{2}$ Conservative Dentistry \& Endodontics, Ph: 9833155591, Email: drdinesh_s@hotmail.com \\ ${ }^{3}$ HOD, Dept. of Conservative Dentistry \& Endodontics \\ Ph: 9820573096, Dr D.Y Patil School of Dentistry Nerul, Navi Mumbai, Maharastra \\ Correspondence Author \\ Dr Priyadarshini Shetty \\ Ph: 9930155591, Email: drpriyadarshini78@gmail.com \\ Address: Flat 201, Millennium Grand Building, Plot 71-B, Sector 11, Kharghar, Navi Mumbai 410210

\begin{abstract}
The objective of this invitro study was to find out if a buccal curve in the palatal roots of maxillary molars affected the clinician's ability to accurately determine working length. 30 extracted intact human maxillary $1^{\text {st }}$ and $2^{\text {nd }}$ molars were sorted by palatal root curvatures as $J$ and $C$ type and angle of curvature was determined using Schneider's method.

Straight line access was made and a No \#20 file was placed into the canal until the tip was visible at the apical foramen under stereomicroscope. The file, tooth and calibration wire were radiographed on one image using the RVG system and the actual (file) and radiographic (tooth) length were determined using the RVG digital ruler. The same No\# 20 file was used with apex locator to record Apex locator length (EL).

Result showed radiographic length $(R L)$ appeared shorter on average than actual length (AL) and apex locator length (EL). A statistically significant difference was seen between the actual length and radiographic length as the degree of curvature increases. Also there was no significant difference between the $J$ type and $C$ type curve. The use of apex locator is a valuable adjunct for working length determination especially in case of palatal root of maxillary molars.
\end{abstract}

\section{INTRODUCTION}

Correct working length determination is the key factor leading to success in root canal treatments. Root canal preparations and filling should not extend beyond the root nor leave uninstrumented areas inside the root canals ${ }^{1}$.

The ideal apical end point of endodontic instrumentation and obturation has been determ- ined to be the cemento-dentinal junction, which occurs on an average $0.5 \mathrm{~mm}$ to $1 \mathrm{~mm}$ short of the apical foramen ${ }^{2}$. In addition, the apical foramen cannot be visualized on a radiograph. Thus the radiographic vertex is often used as a measuring point clinically, though studies have shown that the radiographic vertex does not always coincide with the apical foramen ${ }^{2,3}$. 
Radiographic working length determination for palatal root of maxillary molars may be difficult because of the super-imposition of anatomic structures (i.e., zygomatic arch, maxillary sinus and a buccal curve in apical one-third of the root). This curvature occurs $55 \%$ of the time in maxillary first molars and $37 \%$ of time in maxillary second molars ${ }^{4}$.

Electronic apex locators (EAL) introduced in 1962 to determine the root length during root canal treatment has become an invaluable tool in modern endodontic practice.

The purpose of the present in-vitro study was to

a) To determine if the presence of a buccal curvature in palatal roots of maxillary molars affected the ability of clinician to accurately determine the working length.

b) To quantitatively determine the relationship between the presence of various degrees of buccal curvature in palatal root of maxillary molars and differences found between radiographic length (RL) and Actual length (AL) and length determined with an apex locator (EL).

c) To determine if the use of an apex locator could play a significant role in working length determination in maxillary molars

\section{MATERIALS AND METHOD}

Thirty extracted intact human maxillary $1^{\text {st }}$ and $2^{\text {nd }}$ molars without apical resorption were selected. Each tooth was radiographed from mesio-distal direction using conventional film (Kodak), using a paralleling technique, constant radiation exposure and developing time. Fig( 1)

Using these radiographs the teeth were then sorted by palatal root curvature into the descriptive categories suggested by Nagy et al into J- type and C- type.( FIG 2).

Teeth were placed into the J-type group he palatal root was straight until the apical 3 to $4 \mathrm{~mm}$ at which level a sharp buccal curvature occurred much like the letter 'J'. (FIG 3A)

Teeth were placed into the $\mathrm{C}$ - type if the palatal root had a gradual curve from the coronal and middle third of the root and extended to the root apex much like the letter ' $\mathrm{C}$ '(FIG 3B).

The radiographs were scanned and the angle of palatal root curvature was determined using Schneider's method using Corel draw11 software.

A flat coronal reference point was created by reducing $3 \mathrm{~mm}$ from the occlusal table with a long straight fissure bur and straight line access was made through each tooth sample.

The coronal and middle portion of the palatal canal was flared with Gates Glidden burs using crown down technique and irrigating with normal saline solution. Canal patency was verified with a \#8 file and each sample was instrumented to the verified apical foramen with a No \#20 file size.

Actual length (AL) and radiographic length (RL) were measured in the following manner.

A No \#20 Niti file was used to represent the AL from the coronal reference plane to the apical foramen. The file was introduced into the palatal root of the tooth until the tip of the file was visible under the stereomicroscope, Fig(4). An endodontic rubber file stop was used to mark the file at its coronal reference then withdraw \& set aside. A calibration device, consisting of a $7 \mathrm{~mm}$ length of rectangular orthodontic arch wire was then prepared and also set aside.

To minimize measurement and magnification variables, each tooth was placed in a buccallingual direction next to No \#20 file and calibration was on a Radiovisiography Sensor (Suniray) .Three objects were placed onto the RVG sensor using a polyvinyl siloxane jig to standardize the angle and distance from the $\mathrm{x}$ ray source. From the image taken and using the digital ruler feature of the RVG system, the palatal root of the tooth was measured from the radiographic apex to the coronal reference point (FIG5A\&B). This tooth measurement represents (RL) Using the digital ruler again the distance from the tip of No \#20 Niti file and edge of endodontic file stop was measured. This represented the (AL). Each RVG image was calibrated using the orthodontic arch wire of known length.(FIG 6). 
The electronic measurement (EAL) was achieved using the ipex apex locator [NSK].

The teeth were placed in contact with $2 \%$ agar gel to simulate the periodontium in a plastic container. Both the specimen and the lip clip of the electrode were glued with acrylic resin to the lid of the container to prevent displacement. (Fig 7) No \#20 Niti file was used according to the manufacturer's instructions. The termination point used in this study was the ' 00 ' on the meter designated by the manufacturer as the Apex.
This electronic length (EL) was measured and noted on the RVG as in the case of RL and AL. All tooth samples and files were measured 3 times using the digital ruler on the RVG system to the nearest length of a mm. Analysis of correlation was used to test for a relationship between the amount of curvature and the AL-RL difference (in $\mathrm{mm})$.

In addition difference in the types of curve ( $\mathrm{J}$ versus C) was compared with the regression analysis

\section{RESULTS:}

Results: - The AL, RL and EL value differences are shown in Table I (mm). P $<0.001$

\begin{tabular}{|c|c|c|c|c|c|c|}
\hline & $\begin{array}{l}\text { Tooth No } \\
\text { Curvature } \\
\text { (degrees) }\end{array}$ & $\begin{array}{c}\text { Actual } \\
\text { length (AL) } \\
\text { mm }\end{array}$ & $\begin{array}{c}\text { Radiographi } \\
\text { c length } \\
(\mathrm{RL}) \mathrm{mm}\end{array}$ & AL-RL mm & $\begin{array}{l}\text { Apex locator } \\
\text { RL (EL) mm }\end{array}$ & $(\mathrm{AL}-\mathrm{EL}) \mathrm{mm}$ \\
\hline 1 & 15.341 & 18.06 & 17.93 & 0.13 & 18.07 & 0.01 \\
\hline 2 & 19.849 & 17.60 & 17.40 & 0.20 & 17.65 & 0.05 \\
\hline 3 & 16.321 & 18.02 & 17.96 & 0.06 & 18.03 & 0.01 \\
\hline 4 & 15.524 & 18.80 & 18.74 & 0.06 & 18.81 & 0.01 \\
\hline 5 & 21.583 & 18.01 & 17.61 & 0.40 & 18.03 & 0.02 \\
\hline 6 & 21.346 & 19.06 & 18.75 & 0.31 & 19.07 & 0.01 \\
\hline 7 & 19.472 & 19.03 & 18.83 & 0.20 & 19.04 & 0.01 \\
\hline 8 & 24.419 & 17.00 & 16.57 & 0.43 & 17.04 & 0.04 \\
\hline 9 & 25.397 & 18.48 & 17.98 & 0.50 & 18.58 & 0.10 \\
\hline 10 & 25.306 & 18.53 & 18.00 & 0.53 & 18.46 & 0.01 \\
\hline 11 & 25.215 & 18.50 & 18.00 & 0.50 & 18.51 & 0.01 \\
\hline 12 & 26.125 & 17.80 & 17.17 & 0.63 & 17.82 & 0.02 \\
\hline 13 & 25.867 & 20.14 & 19.61 & 0.53 & 20.15 & 0.01 \\
\hline 14 & 28.877 & 17.00 & 16.37 & 0.63 & 17.01 & 0.01 \\
\hline 15 & 31.036 & 18.21 & 17.62 & 0.59 & 18.23 & 0.02 \\
\hline 16 & 32.618 & 18.24 & 17.68 & 0.56 & 18.25 & 0.01 \\
\hline 17 & 34.185 & 18.05 & 17.69 & 0.36 & 18.07 & 0.02 \\
\hline 18 & 33.346 & 19.10 & 18.49 & 0.61 & 19.11 & 0.01 \\
\hline 19 & 33.541 & 17.03 & 16.41 & 0.62 & 17.05 & 0.02 \\
\hline 20 & 36.675 & 17.67 & 18.57 & 1.10 & 17.68 & 0.01 \\
\hline 21 & 36.438 & 20.00 & 19.20 & 0.80 & 20.01 & 0.01 \\
\hline 22 & 34.916 & 17.89 & 17.21 & 0.68 & 17.82 & 0.02 \\
\hline 23 & 39.126 & 20.13 & 19.37 & 0.76 & 20.14 & 0.01 \\
\hline 24 & 39.150 & 19.12 & 18.36 & 0.76 & 19.13 & 0.01 \\
\hline 25 & 42.516 & 18.04 & 17.51 & 0.53 & 18.05 & 0.01 \\
\hline 26 & 44.312 & 18.16 & 17.40 & 0.76 & 18.18 & 0.02 \\
\hline 27 & 50.777 & 19.95 & 18.85 & 1.10 & 20.00 & 0.05 \\
\hline 28 & 55.938 & 18.26 & 16.86 & 1.40 & 18.31 & 0.05 \\
\hline 29 & 56.321 & 17.55 & 16.05 & 1.50 & 17.55 & 0.05 \\
\hline 30 & 57.120 & 19.02 & 18.01 & 1.10 & 19.07 & 0.05 \\
\hline
\end{tabular}

According to results derived as shown in Table I, RL appeared shorter on average than the AL \& EL. The Difference between actual length (AL) and Apex locator Length (EL) was not found to be statistically significant. 


\section{JMSCR Vol||05||Issue||03||Page 18484-18489||March}

\section{GRAPH I}

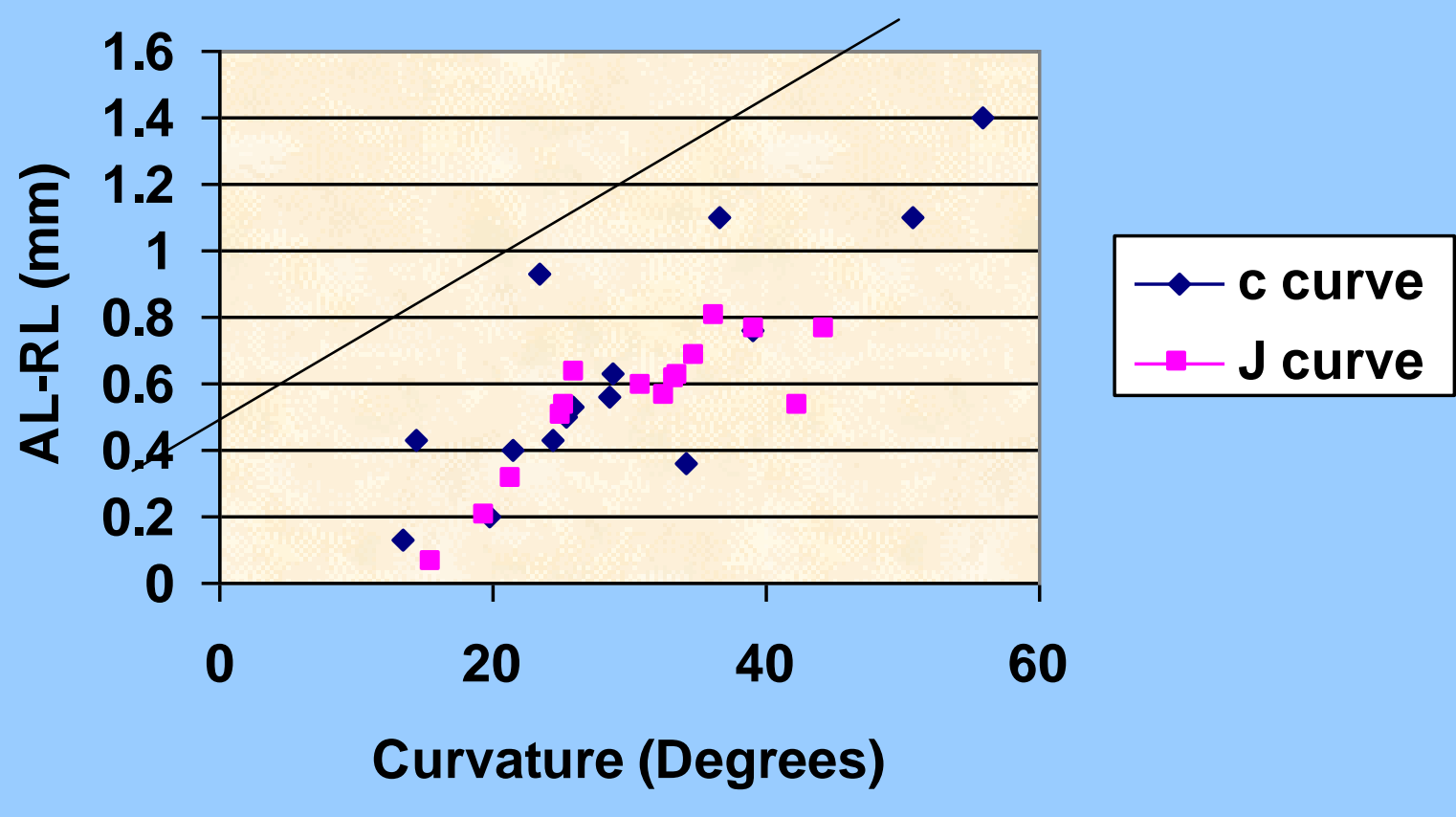

The relationship between curvature and the ALRL difference was a line through zero with a significant slope $(\mathrm{p}<0.001)$.There was a signifycant difference between actual length and Radiographic length in canal curvatures larger

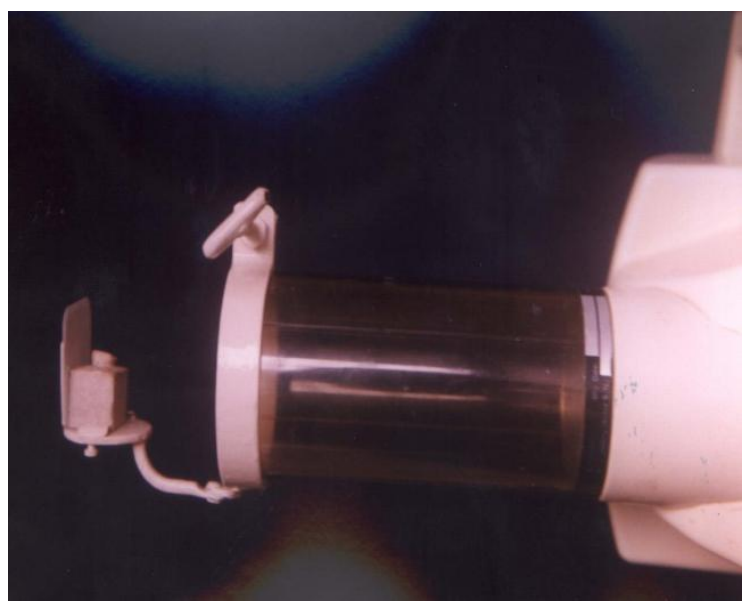

FIG 1: Each tooth was radiographed from mesiodistal direction using paralleling technique. than 25 degrees. As the degree of curvature increased the difference between AL \& RL also increased. There was no canal type curvature interaction thereby indicating no difference between the $\mathrm{J}$ type and $\mathrm{C}$ type of curve.

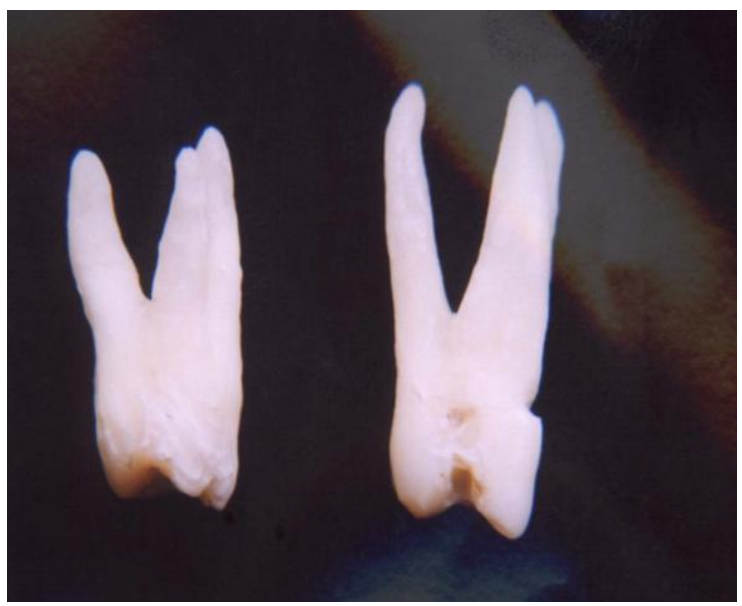

FIG 2: C type J type curvatures of palatal roots 


\section{JMSCR Vol||05||Issue||03||Page 18484-18489||March}

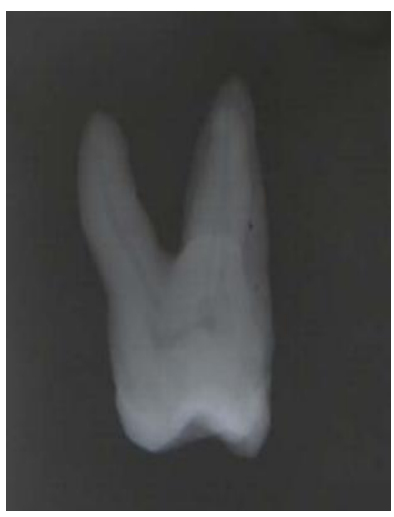

(A)

Fig.3 (A) C type canal and 3 (B) J type canal

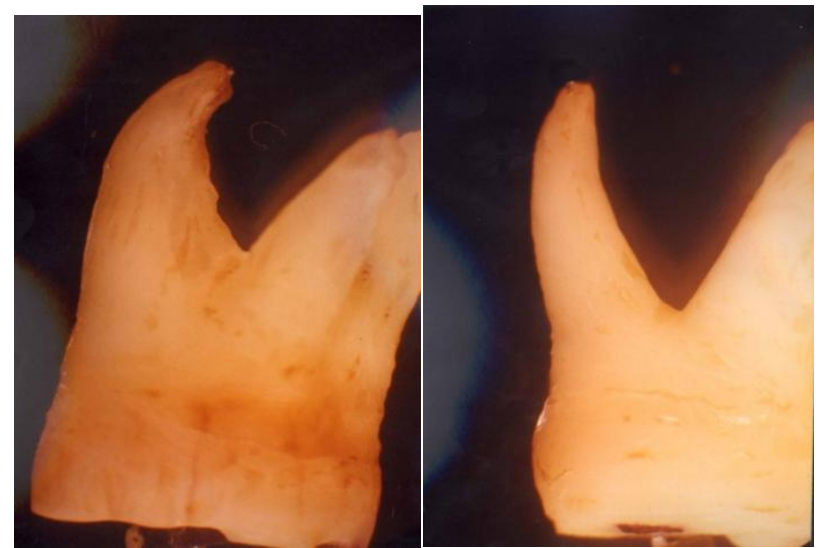

Fig 4: Tip of File Visible Under Stereomicroscope

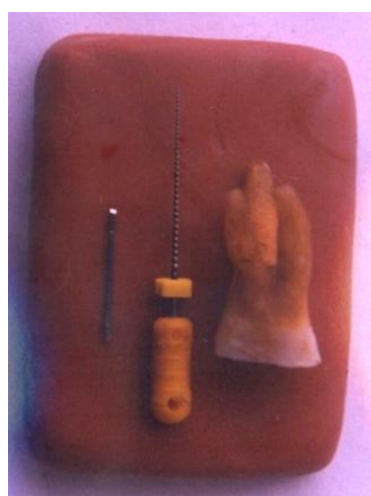

A

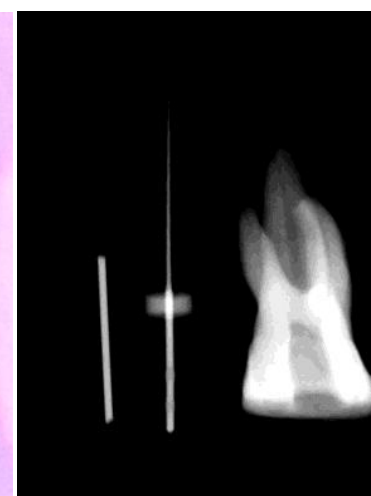

B
FIG 5 (A): Calibration wire, file,tooth placed on polyvinylsiloxane jig 5 (B): Image on RVG.

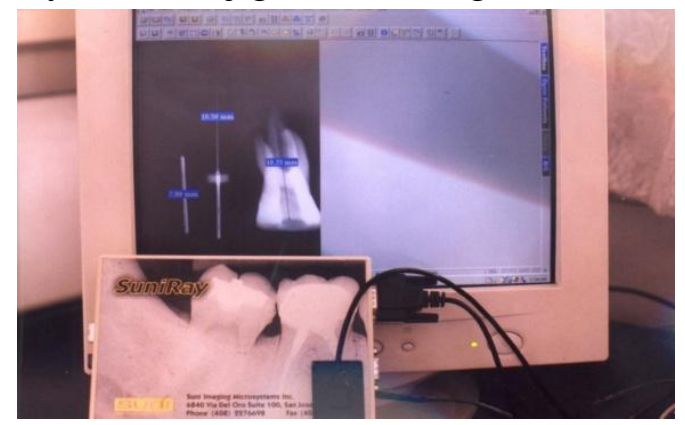

FIG 6: Measurements taken using digital ruler feature of the RVG

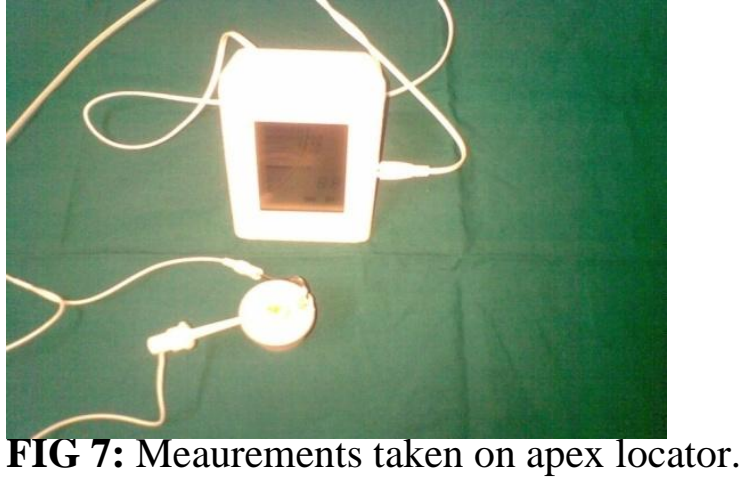

\section{DISCUSSION}

In this invitro study, extracted maxillary molars were used to find out if a buccal curve in the palatal root affected the ability of a clinician to accurately determine working length.

Although many studies have looked at the mesiobuccal root of maxillary molars, the palatal root has not received as much attention in regard to palatal anatomy for canal instrumentation and obturation.

Schneider's angle was used to qualitatively determine the degree of canal curvature present and determine if a relationship existed between curvature and AL-RL difference and AL-EL difference. Results showed a statistically signifycant difference between Radiographic working lengths as the degree of curvature increases.

RL also appeared on the average to be shorter than the ALof curvature, irrespective of the type of $\mathbf{J}$ or $\mathrm{C}$ curve, this finding supports the work of Stein and Corcoran (1992) ${ }^{5}$. This findings may be in part due to a) The curvature in the palatal root is typically viewed in the buccal-lingual orientation creating a geometric illusion that makes the viewed length appear shorter than the actual length.

b) Also as the apical foramen does not always coincide with the radiographic vertex.

Thus using only radiographic length to determine working length may produce over instrumentation into the periradicular tissues. These findings are in conjunction with the findings of El Ayouti et $\mathrm{al}^{6}$. RVG was used with digital ruler feature. The purpose of keeping all three objects(file, tooth and calibration unit) on one sensor was to eliminate 
operator variance and bias in measurement procedures.

Larger files deviated less from the known lengths on RVG images, so large size No \# 20 was selected to measure $\mathrm{AL}$ and all measurements were taken in a positive/negative zoom mode using the RVG system.

The use of electronic apex locator to determine working length has gained in popularity ${ }^{7}$. The apical limit used was the apical foramen. The apex locator reading was nearly same as that of the actual length of the palatal root. This study also agrees with others (Usen et al 2007, 2008) ( $^{8,9}$ that Electronic apex locators are more accurate then radiographs and greatly reduce the risk of instrumenting and filling short or beyond the canal terminus.

\section{CONCLUSION}

The ability of a clinician to accurately determine working length based solely on radiographic interpretation may be impaired because of the significant buccal curvature in the palatal roots of maxillary molars.

As the degree of curvature increases the discrepancy between AL and RL and AL and EL will increase significantly.

There are limitations in using radiographs as a sole means of determining working length and hence must be combined with adjunts such as electronic apex locators

The electronic apex locator with its high degree of accuracy \& ease of working is highly recommended adjunct in working length determination in case of palatal root of maxillary molars

\section{REFERENCES}

1. V.P.Vieyra, J.Acosta, J.M Mondaca. Comparision of working length determi-nation with radiographs and two electronic apex locators. Int End Journal 2010;43: 16-20.

2. Kuttler Y. Microscopic investigation root apices. J Am Dent Asso 1955;50: 544-52.
3. Dummer PMH. The position and topography of the apical canal constriction and apical foramen. Int End Journal1984;17: 192-8.

4. Prieda F. radiogarphic investigation of the mesiobuccal root of maxillary permanent first molar. Oral Sur Oral Med Oral Pathol 1973;33: 251-3.

5. Stein TJ, Corcoran J F, Radiographic "working length" revisited. Oral Surg Oral Med Oral Pathol 1992;74: 796-800.

6. El Ayouti, Weiger R. Frequency of overinstrumentation with an acceptable radiographic working length. JOE 2001;27: 49-52.

7. Cunha D'assuncao. The ability of two apex locators to locate the apical foramen: an invitro study. JOE 2006;32: 560-562.

8. Usur, Sadik B. Accuracy of the apex locating function of TCM endo $\mathrm{V}$ in simulated conditions: a comparision study. Oral Surg Oral Med Oral Path 2007;103: 73-76.

9. Usun, Dummer. Accuracy of two root canal length measurement devices integrated into rotary endodontic motors when removing guttapercha from root filled teeth. Int Endo Journal 2008;41: 725-732. 